\title{
Antibiotic susceptibility pattern of staphylococcus aureus and methicillin-resistant staphylococcus aureus in a tertiary care hospital
}

\author{
Bhatt CP ${ }^{1}$, Karki BMS'2, Baral B ${ }^{3}$, Gautam S ${ }^{3}$, Shah A³, Chaudhary A ${ }^{3}$ \\ ${ }^{1}$ Department of Microbiology, Kathmandu Medical College Public Limited, Sinamangal Kathmandu Nepal \\ ${ }^{2}$ Department of Microbiology, Kist Medical College, Gwarkhu, Nepal \\ ${ }^{3}$ Department of Microbiology, Nobel College, Kathmandu, Nepal
}

\author{
Keywords: \\ Nosocomial infection; \\ MRSA; \\ Staphylococcus aureus.
}

\begin{abstract}
Background: Methicillin resistant Staphylococcus aureushas emerged as one of the most important nosocomial pathogens. It invokes a tremendous financial burden and enhanced morbidity and mortality due to difficult to treat systemic infections.Aim of this study was to determine antibiotic susceptibility pattern of Staphylococcus aureus and Methicillin resistant Staphylococcus aureus.

Materials and Methods: Different clinical specimens were collected and processed for routine culture and antibiotic sensitivity test by standard microbiology techniques.

Results: Out of 1173 samples received for microbiological examination, 100 were found to be S. aureus with 19\% cases were Methicillin resistant Staphylococcus aureus (MRSA). Fourteen MRSA were found from inpatient and 5 were from outpatient. MRSA was found higher in female than male and maximum number (31.5\%) was found in age group 0-10 years. Staphylococcus aureus was 100\% sensitive to Vancomycin followed by Amikacin (90\%), Gentamycin (83\%), and tetracycline (81\%). On urine isolates Nitrofurantoin(91.6\%) was drug of choice. All the isolates were resistant to Penicillin G. In case of Methicillin resistant Staphylococcus aureus showed 100\% sensitive to Vancomycin followed by Amikacin (84.2\%), Tetracycline (63.1\%), Ciprofloxacin (42\%) and Gentamycin (36.8\%). Among urine isolates Nitrofutantoin showed $87.5 \%$ sensitive followed by Norfloxacin (75\%).

Conclusion: Methicillin resistant Staphylococcus aureus was found 19\% of Staphylococcus aureus isolates. It was most common in females, hospitalized patients and young age group. Vancomycin seems to be drug of choice followed by Amikacin. It would be helpful to formulating and monitoring the antibiotic policy and ensure proper empiric treatment.
\end{abstract}

\section{INTRODUCTION}

Staphylococcus aureus (S. aureus) is important cause of communityand hospitalacquired infections. Infections

\section{Correspondence:}

Dr. Chandra Prakash Bhatt

Associate Professor, Department of Microbiology

Kathmandu Medical College Public Limited

E-mail:drcpbhatt@yahoo.com,drcpbhatt@gmail.com caused by Methicillin or Oxacillin resistant $\mathrm{S}$.aureus (MRSA) are mainly nosocomial and are increasingly reported from many countries worldwide. ${ }^{1}$ As MRSA strain are frequently resistant to many different classes of antimicrobial drugs, second-and third line antimicrobial resistance is a growing concern. ${ }^{2}$ Methicillin was first introduced in human medicine in the 1960s for the treatment of infections caused by Penicillin's resistant S. aureus, but 
within a few years, MRSA emerged. ${ }^{3}$ Methicillin resistance in staphylococci is mediated by the mecAgene, which encodes for the penicillin-binding protein 2A (PBP2A) resulting in reduced affinity for the beta-lactam antibiotics including the penicillinase-resistant penicillin. MRSA has become a major hospital pathogen in human medicine. ${ }^{4}$

However, Vancomycin resistant S.aureus is not widely seen even though a low level resistance to Vancomycin is being reported. ${ }^{5}$ The risk factors which contribute to MRSA are excessive antibiotic usage, prolonged hospitalization, intravascular catheterization and hospitalization in intensive care unit.

The prevalence rate of MRSA is various in different countries such as $1-5 \%$ in Northern Europe, $5-30 \%$ in Southern Europe, 5-40 \% in Asian countries and 10-50\% in USA and in UK. The problems of treating such infections are mainly due to the multi-drug resistant nature of MRSA. The prevalence of MRSA in Nepal is reported to be $15-69 \%$ from different tertiary care hospitals. ${ }^{6-8}$

The emergence of antibiotic resistant bacteria constitutes a major problem in antibiotic therapy. This could be attributed to unrestricted use of antibiotics in a particular environment. The present study aim was to know antibiotic sensitivity pattern of Staphylococcus aureus isolates in order to utilize the information obtained and formulate antibiotic policy and appropriate control measures.

\section{MATERIALS AND METHODS}

A prospective study was conducted at Microbiology Laboratory of Kist Medical College, Lalitpur during August 2011 to November 2011 included all patients both outpatient and inpatient. The research objective and methods were explained to the patients and informed consent was taken from each patient before collection of specimen.

In this study 1173 different clinical specimens(urine, blood, pus, sputum, swab, fluid/CSF and tips)were collected for routine culture and antibiotic susceptibility testing and were processed according to the standard microbiology laboratory techniques. The sample collected was evaluated in terms of its acceptability, proper labeling (full name, age, sex, serial number of the patient, date of collection).

The specimens were cultured on blood agar and MacConkey agar plates and incubated aerobically at $37^{\circ} \mathrm{C}$ for 24 hours. Staphylococcus aureus was identified by standard microbiology test such as colonies characteristics, gram staining reaction, biochemical tests such as catalase test, coagulase test and mannitol salt fermentation test.Antibiotic sensitivity of the isolates was performed according to the guidelines given by the NCCLS recommended KirbyBauer sensitivity testing method.The antibiotics which were used based on the standard protocol of the hospital and the department policies. The antibiotic discs used for the susceptibility tests were from Hi-Media Laboratories Pvt. Limited. Different antibiotics used for Staphylococcus aureus are as follows: Penicillin (10 units), Ampicillin $(10 \mu \mathrm{g})$, Vancomycin $(30 \mu \mathrm{g})$, Amikacin $(30 \mu \mathrm{g})$, Cotrimoxazole $(1.25 / 23.75 \mu \mathrm{g})$, Ciprofloxacin $(5 \mu \mathrm{g})$, Gentamycin $(30 \mu \mathrm{g})$, Tetracyclin $(30 \mu \mathrm{g})$, Erythromycin $(15 \mu \mathrm{g}), *$ Nalidixic Acid $(30 \mu \mathrm{g})$, *Norfloxacin $(10 \mu \mathrm{g})$, *Nitrofurantoin $(300 \mu \mathrm{g})$, * used only for urine isolates. Screening of MRSA was performed according to the guidelines given by the Clinical and Laboratory Standards Institute 2011.

Data was analyzed by EPI-Info version 3.3.2, document version 8.08 updated Sept 2005 and presented by tables and diagrams.

\section{RESULTS}

Out of 1173 samples 328 (27.9\%) were culture positive. Among them $46.3 \%$ were urine, $32.9 \%$ were blood, $11.5 \%$ were pus, $4.5 \%$ were sputum, $3.1 \%$ were swab, $0.25 \%$ were fluid/CSF and $1.5 \%$ were tips. Out of 328 cultures positive cases, prevalence of S.aureuswere 100 (30.4\%) and others were 228 (69.5\%). Among 100 isolates of S.aureus, 19 were found to be Methicillin resistant S.aureus. The isolates of S.aureus and MRSA was higher in ward patient (54\%) and $(73.6 \%)$ than OPD $(46 \%)$ and $(26.3 \%)$ respectively. Gender wise distribution of S. aureus and Methicillin resistant Staphylococcus aureus was found higher in female than male. Agewise distribution of S.aureus and MRSA is shown in Table 1 in which Staphylococcus aureus was most prevalent in 51-60 years old group and MRSA in children (0-10 years). Antibiotic susceptibility pattern of both $\mathrm{S}$. aureus and MRSA is shown in Table 2.

\section{DISCUSSION}

Despite the introduction of antimicrobial therapy and the recent improvements of medical services, MRSA is recognized as a major cause of nosocomial infections which result in significant morbidity and mortality rates.S.aureus is a very common cause of infection in hospitals and is most liable to infect new born babies, surgical patients, old and malnourished persons and patients with diabetes and other chronic diseases. ${ }^{9}$

A total of 1173 samples were processed, out of them 328 were positive for bacterial growth. In culture positive cases S. aureus accounted for $30.4 \%$ (100). The number of S. aureus isolated was high in ward (54\%) than OPD (46\%) and isolates were high in females than males. Out of $100 \mathrm{~S}$. aureus isolates 19\% isolates were found to be Methicillin resistant S. aureus. Out of that 8 MRSA were isolated from urine, 6 from blood and 5 from pus. In contrast to our study, Mahmood et al included 265 cases of which 102 (38.4\%) were from sputum, 76 (28.6\%) from IV catheters/ ETT/suction tips etc $65(24.5 \%)$ from pus/wound swabs, 
Table 1: Age wise distribution of Staphylococcus aureus and Methicillin resistant S. aureus

\begin{tabular}{ccccccc}
\hline $\begin{array}{c}\text { Age } \\
\text { group }\end{array}$ & \multicolumn{3}{c}{ Staphylococcus aureus } & \multicolumn{3}{c}{$\begin{array}{c}\text { Methicillin resistant S. } \\
\text { aureus }\end{array}$} \\
\hline & Male & Female & $\%$ & Male & Female & $\%$ \\
$0-10$ & 16 & 8 & $24 \%$ & 5 & 1 & $31.5 \%$ \\
$11-20$ & 3 & 14 & $17 \%$ & & 5 & $26.3 \%$ \\
$21-30$ & 6 & 12 & $18 \%$ & & 3 & $15.7 \%$ \\
$31-40$ & 5 & 7 & $12 \%$ & & 2 & $10.5 \%$ \\
$41-50$ & & 10 & $10 \%$ & & 2 & $10.5 \%$ \\
$51-60$ & 10 & 9 & $19 \%$ & & 1 & $5.2 \%$
\end{tabular}

17 (6.4\%) from blood and 5 (1.8\%) from urine cultures. ${ }^{10}$ Another study conducted by Vaez et al rate of MRSA strains isolation in wound and sputum were higher than other samples with $42.9 \%$ and $40 \%$ respectively. ${ }^{11}$ This is consistent with the reports fromTahnkiwale reported 19.5\% of the total isolates of the S.aureus were MRSA. ${ }^{12}$

The patients between 0-10 age group showed the maximum positive growth for S. aureus (24\%) followed by age group 51-60 (19\%). The patients between 0-10 age group showed the maximum MRSA (31.5\%), followed by age group 11-20 (26.3\%). Age group 0-10 tends to have higher colonization rates, probably because of their frequent contact with respiratory secretions and may be due to sanitary reason. It is believed that their immunity is not properly developed at this stage to cope with bacterial infections hence they are vulnerable and easily infected especially when hospitalized. The older children have also been observed to be more active than adults during their interaction with their playmates and while playing for hours, come in contact with various objects. In this process, they become a target to ubiquitous bacteria such as S. aureus.In contrast to this study Mahmood et al reported that majority of patients $170(64.1 \%)$ were in age group $41-80$ years. ${ }^{10}$

Finding of this study showed that S. aureus were found higher in female $60 \%$ than male $40 \%$ and MRSA were also found higher in female $73.6 \%$ than male $26.3 \%$. Male and Female ratio of this distribution was 2:3. In contrast to this study MRSA predominance was observed in males 155 $(58.5 \%)$ than female $110(41.5 \%)$, Male to female ratio was 1:0.7.10Another study performed by Khanal et althe rate was significantly higher among males $(75 \%)$ than females $(25 \%)$. MRSA infection was higher among patients above 30 years of age. ${ }^{13}$

Inpatients department showed maximum number of $\mathrm{S}$. aureus (54\%) and outpatient department showed (46\%). Out of 19 MRSA (73.6\%) were from inpatient department and $(26.3 \%)$ were from outpatient department. This difference could be due to prolonged hospital stay, instrumentation and other invasive procedures and S.aureus is one of the major organisms causing hospital acquired infection. Study conducted by Sanjhana et al showed that (62.3\%) Methicillin resistant S. aureus were isolated from inpatient departments and $(37.7 \%)$ were from outpatients, which is comparable with our study. ${ }^{14}$

Antibiotic susceptibility pattern of S. aureus showed that Vancomycin was $100 \%$ sensitive, followed by Amikacin $90 \%$, Gentamycin 83\%, Tetracycline 81\%, Ciprofloxacin

Table 2: Antibiotic susceptibility pattern of S. aureus and Methicillin resistant S. aureus

\begin{tabular}{|c|c|c|c|c|c|c|}
\hline \multirow[b]{2}{*}{ Antibiotics } & \multicolumn{3}{|c|}{ Staphylococcus aureus } & \multicolumn{3}{|c|}{ Methicillin resistant S. aureus } \\
\hline & Sensitive & Intermediate & Resistance & Sensitive & Intermediate & Resistance \\
\hline Penicillin & $0(0 \%)$ & $0(0 \%)$ & $100(100 \%)$ & $0(0 \%)$ & $0(0 \%)$ & $19(100 \%)$ \\
\hline Ampicillin & $22(22 \%)$ & $3(3 \%)$ & $75(75 \%)$ & $2(10.5 \%)$ & $3(15.7 \%)$ & $14(73.6 \%)$ \\
\hline Vancomycin & $100(100 \%)$ & $0(0 \%)$ & $0(0 \%)$ & $19(100 \%)$ & $0(0 \%)$ & $0(0 \%)$ \\
\hline Amikacin & $90(90 \%)$ & $6(6 \%)$ & $4(4 \%)$ & $16(84.2 \%)$ & $0(0 \%)$ & $3(15.7 \%$ \\
\hline Cotrimoxazole & $58(58 \%)$ & $13(13 \%)$ & $29(29 \%)$ & $6(31.5 \%)$ & $1(5.2 \%)$ & $12(63.1 \%)$ \\
\hline Ciprofloxacin & $59(59 \%)$ & $22(22 \%)$ & $19(19 \%)$ & $8(42.1 \%)$ & $2(10.5 \%)$ & $9(47.3 \%)$ \\
\hline Gentamycin & $83(83 \%)$ & $2(2 \%)$ & $15(15 \%)$ & $7(36.8 \%)$ & $1(5.2 \%)$ & $11(57.8 \%)$ \\
\hline Tetracyclin & $81(81 \%)$ & $5(5 \%)$ & $14(14 \%)$ & $12(63.1 \%)$ & $2(10.5 \%)$ & $5(26.3 \%)$ \\
\hline Erythromycin & $31(31 \%)$ & $29(29 \%)$ & $40(40 \%)$ & $1(5.2 \%)$ & $1(5.2 \%)$ & $17(89.4 \%)$ \\
\hline *Nalidixic Acid & $3(12.5 \%)$ & $0(0 \%)$ & $21(87.5 \%)$ & $0(0 \%)$ & $0(0 \%)$ & $8(100 \%)$ \\
\hline *Norfloxacin & $12(50 \%)$ & $0(0 \%)$ & $12(50 \%)$ & $6(75 \%)$ & $0(0 \%)$ & $2(25 \%)$ \\
\hline *Nitrofurantoin & $22(91.6 \%)$ & $0(0 \%)$ & $2(8.3 \%)$ & $7(87.5 \%)$ & $1(12.5 \%)$ & $0(0 \%)$ \\
\hline
\end{tabular}


59\%, Cotrimoxazole 58\%, Erythromycin 31\%, Ampicillin $22 \%$ and all the isolates were resistant to Penicillin G. Nitrofurantoin, Norfloxacin and Nalidixic acid were used for urine isolates and showed sensitivity of $91.6 \%, 50 \%$ and $12.5 \%$ respectively. Study conducted by Khanal et al showed that resistance pattern of $\mathrm{S}$. aureus to different antibiotics, Erythromycin $75.3 \%$, Ciprofloxacin $70 \%$ and Vancomycin $21 \%$. That study indicates the emergence of Vancomycin resistant S. aureus (VRSA). ${ }^{13}$ Similar study performed by Baral et al S. aureus showed susceptible to Tetracycline (94.3\%), Erythromycin (89\%), Ciprofloxacin $(88.3 \%)$ and Gentamicin (78\%). Sixty four percent of isolates were found to be resistant to Co-trimoxazole and 26\% were Methicillin resistant (MRSA). Nearly half (52. $6 \%$ ) of $\mathrm{S}$ aureus showed resistance to Penicillin. All the isolates were susceptible to Vancomycin. ${ }^{16}$ In study done by Vaez et al resistance pattern to Penicillin, Ampicillin, Nalidixic acid, Erythromycin, Ciprofloxacin, Gentamicin, Tetracycline and Vancomycin was 97.3\%, 97.3\%, 53\%, $29.2 \%, 22.7 \%, 20.5 \%, 31.4 \%$ and $0 \%$ respectively. ${ }^{11}$ These studies are consistent with our finding.

Antibiotic susceptibility pattern of Methicillin resistant S. aureus showed that Vancomycin was $100 \%$ sensitive followed by Amikacin 84.2\%, Tetracycline $63.1 \%$, Ciprofloxacin 42.1\%, Gentamycin 36.8\%, Cotrimoxazole $31.5 \%$, Ampicillin 10.5\%, and Erythromycin 5.2\%. In urine isolates Nitrofurantoin showed $87.5 \%$ sensitive followed by Norfloxacin $75 \%$ and all the isolates are resistant to Nalidixic acid. This is consistent with the reports from Mahmood et al antimicrobial sensitivity pattern of MRSA showed $28.7 \%$ to Ciprofloxacin, $37.5 \%$ to Gentamycin, $27.5 \%$ to Erythromycin, $8 \%$ to Penicillin and $100 \%$ to Vancomycin. ${ }^{10}$

\section{CONCLUSION}

In this study Methicillin resistant S.aureus was found 19\% of the Staphylococcus aureus positive cases. MRSA was common in female, admitted patients and lower age group. For treatment of S. aureus infection Vancomycin (100\%) was the drug of choice followed by Amikacin (90\%), Gentamycin (83\%), and Tetracycline (81\%). On urine isolates Nitrofurantoin( $91.6 \%)$ was drug of choice. All the isolates were resistant to Penicillin G. In case of MRSA Vancomycin (100\%) seems to be drug of choice followed by Amikacin (84.2\%). This study highlights the need for continuous surveillance of antibiotic sensitivity pattern of S.aureus and (MRSA). It would be helpful to formulating and monitoring the antibiotic policy and ensure proper empiric treatment.

\section{REFERENCES}

1. Lowy FD. Staphylococcus aureus infections. NEngl J Med 1998;339:520-32. CrossRef

2. Teover FC, Biddle JW, Lancaster MV. Increasing resistance to vancomycin and other glycopepties in Staphylococcus aureus.Emerg Infect Dis 2001;7:327-32. CrossRef
3. Cookson B, Schmitz F, Fluit A. Introduction In: A.C. Fluit and F.J. Schmitz, Editors, MRSA. Current Perspectives, Caister Academic Press, Wymondham, 2003. pp1-9

4. Rohrer S, Bischoff M, Rossi J, Berger-Bächi B. Mechanisms of methicillin resistance. In: A.C. Fluit and F.-J. Schmitz, Editors, MRSA.Current Perspectives, Caister Academic Press, Wymondham, 2003, pp31- 53 .

5. Assadullah S, Kakru D, Thoker M, Bhat F, Hussai N, Shah A. Emergence of low level vancomycin resistance in MRSA. Indian J Med Microbiol 2003;21:196-8. PMid:17643019

6. Tiwari HK, Sapkota D, Das AK, Sen MR. Assessment of different tests to detect methicillin resistant Staphylococcus aureus. Southeast Asian J Trop Med Public Health 2009;40:801-6. PMid:19842418

7. Kumari N, Mahopatra TM, Singh YI. Prevalence of MethicillinResistant Staphylococcus aureus (MRSA) in a Tertiary-Care Hospital in Eastern Nepal. J Nepal Med Assoc2008;47:53-6. PMid:18709031

8. Subedi $\mathrm{S}$ et al. Antimicrobial susceptibility patterns of clinical isolates of Staphylococcus aureus in Nepal. ClinMicrobiol Infect 2005;11:235-7. CrossRef

9. Tuo P, Montobbio G, Vallarino R, Tumolo M, Calero MG, Massone MA. Nosocomial Staphylococci in a neonatal and pediatric intensive care unit. Pediatr Med Chir. 1995;17:117-22. PMid:7610072

10. Mahmood K, Tahir T, Jameel T, Ziauddin A, Aslam HF. Incidence of Methicillin-resistant Staphylococcus aureus (MRSA) Causing Nosocomial Infection in a Tertiary Care Hospital. Annals of King Edward Medical University 2010;16:2.

11. Vaez H, Tabaraei A, Moradi A and Ezzat A. et al.Evaluation of methicillin resistance Staphylococcus aureusisolated from patients in Golestan province-northof Iran. African Journal of Microbiology Research 2011;5,pp432-6.

12. Tahnkiwale SS, Roy S, Jalgaonkar SV. Methicillin resistance among isolates of Staphylococcus aureus: Antibiotic sensitivity pattern \&phage typing. Indian J Med Sci 2002;56:330-4. PMid:12645169

13. Khanal LK and Jha BK. Prevalence of Methicillin resistant Staphylococcus aureus (MRSA) among skin infection cases at a hospital in Chitwan. Nepal, Nepal Med Coll J 2010;12:224-8.

14. Sanjana RK, ShahR,ChaudharyN, SinghYI. Prevalance and antimicrobial susceptibility pattern of methicillin resistanatStaphylococccusaureus (MRSA) in CMS-teaching hospital: a preliminary report. Journal of College of Medical Sciences Nepal 2010;6:1-6. CrossRef

15. Baral R, Khanal B, Acharya A. Antimicrobial susceptibility patterns of clinical isolates of Staphylococcus aureus in Eastern Nepal. Health Renaissance 2011 2:78-82. 